\title{
Sciences de l'homme et patrimoines naturels
}

Quelques jalons historiques et théoriques

\section{Isac Chiva}

\section{OpenEdition}

Journals

Édition électronique

URL : https://journals.openedition.org/tc/3939

DOI : $10.4000 /$ tc.3939

ISSN : 1952-420X

Éditeur

Éditions de l'EHESS

Édition imprimée

Date de publication : 31 décembre 2008

Pagination : 16-39

ISSN : 0248-6016

Référence électronique

Isac Chiva, «Sciences de l'homme et patrimoines naturels », Techniques \& Culture [En ligne], 50 | 2008, mis en ligne le 31 décembre 2010, consulté le 29 septembre 2022. URL : http://

journals.openedition.org/tc/3939; DOI : https://doi.org/10.4000/tc.3939 


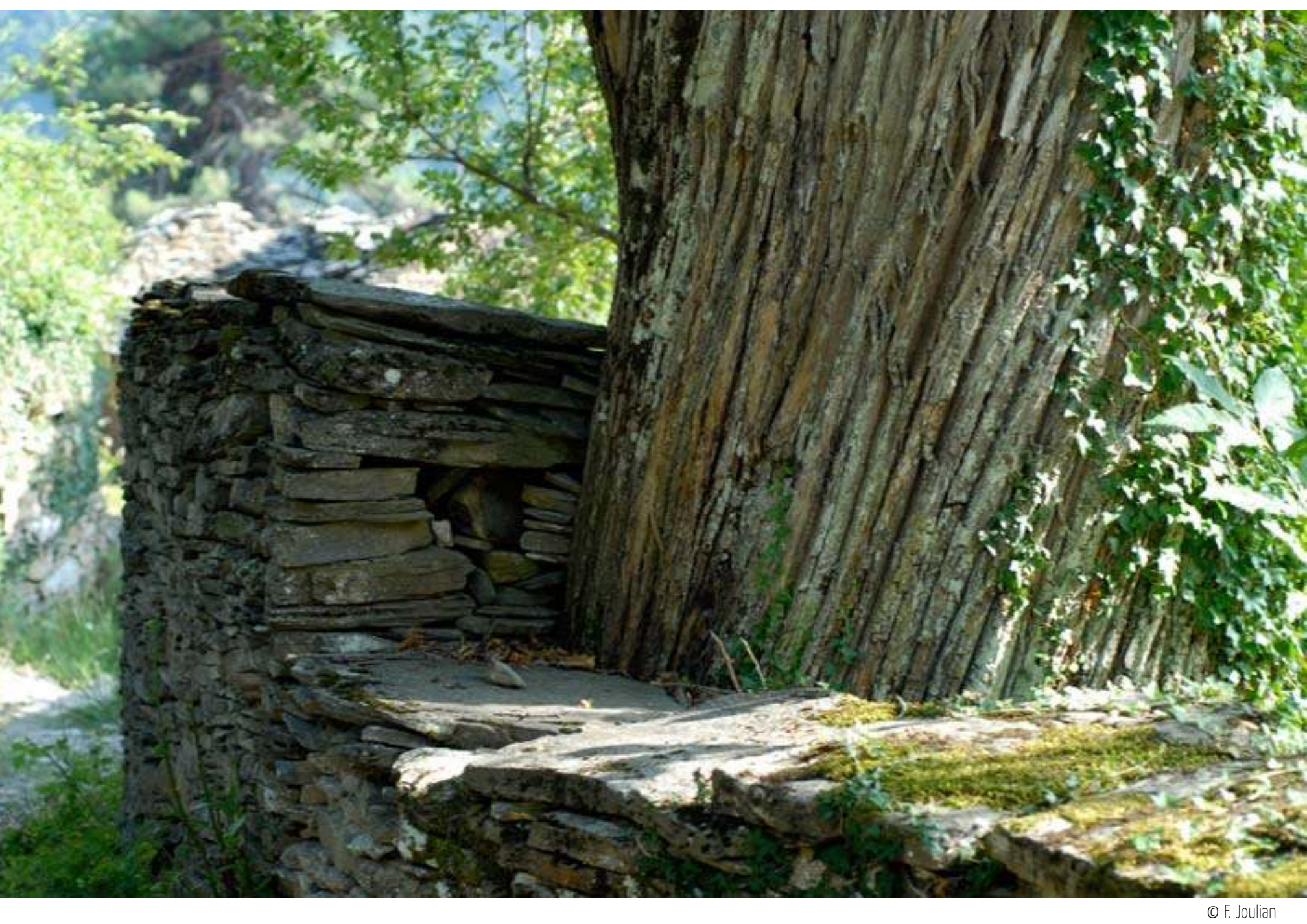


Isac Chiva

Laboratoire d'anthropologie sociale

EHESS
Les natures de l'homme - I

Techniques E Culture 50, 2008 : 16-39

\section{SCIENCES DE L'HOMME ET PATRIMOINES NATURELS}

\section{Quelques jalons historiques et théoriques}

\section{Où l'auteur revisite de l'intérieur l'histoire croisée de la création des parcs naturels et du patrimoine culturel.}

Pour aborder le sujet de ce premier numéro de la nouvelle série de la revue Techniques $\&$ culture sur « les natures de l'homme », je ne pourrai, en ruraliste et en ethnologue du proche, du familier, de l'espace français et européen, que rappeler ce que j'ai toujours préconisé et pratiqué : une démarche multidisciplinaire. Celle-ci est, avant tout, imposée par mon objet premier et constant: la société rurale, en son espace, ses composantes, ses échelles multiples. Elle est aussi consubstantielle au développement historique et épistémologique de l'école française et européenne des études rurales.

Mon premier travail de terrain, au début des années 1950, s'est déroulé en Sologne: j'y ai découvert un système régional, et ce caractère de système m’a immédiatement sauté aux yeux. Pour le comprendre, la prise en compte de l'histoire et de la composition sociale et culturelle ne suffisait pas. Les caractéristiques géographiques, géologiques, pédologiques, climatiques de cet espace plat, riche en étangs et en bois créés par la main de l'homme, s'imposaient à l'attention de l'ethnologue. D'emblée j'ai dû, malgré ma formation naturaliste limitée, assimiler l'analyse écologique 


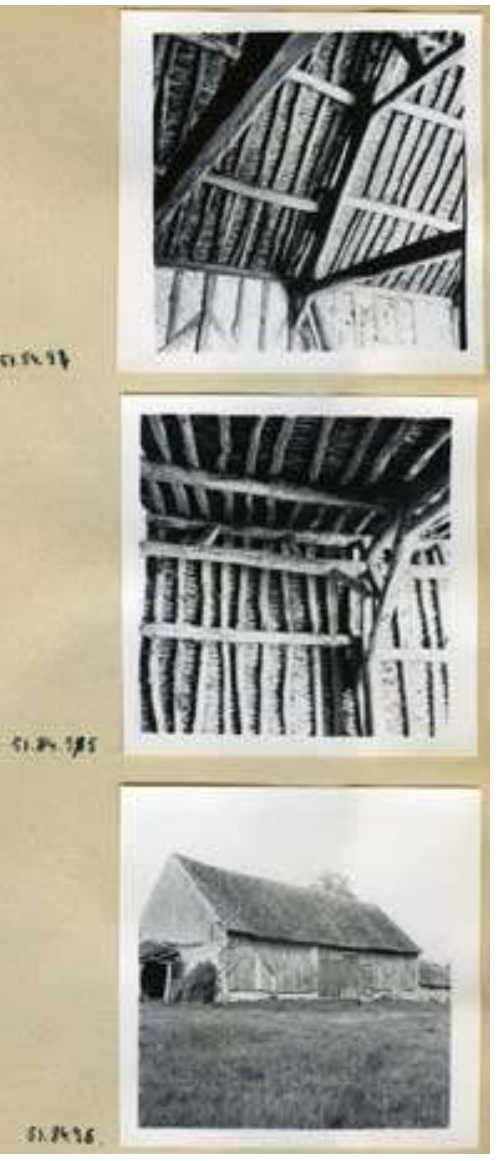

St Viatre, Loir-et-Cher, 1951, I. Chiva de cet ensemble régional, proposée alors par Braun-Blanchet, tout en prenant en compte simultanément les activités cynégétiques et halieutiques qui se superposaient, dans cet espace très particulier, à une agriculture passablement archaïque. L'espace solognot imposait la lecture simultanée du micro-local, de la ferme avec ses locatures, du terroir villageois avec son noyau aggloméré, des superpositions de régimes fonciers: de vastes propriétés souvent entre les mains de parisiens, un fermage différencié, des locations de chasses, des étangs tantôt pêchés, tantôt cultivés. Tout naturellement, la première expression de ce travail d'analyse fut multidimensionnelle et prit une forme muséographique. Avec comme thème " Terroirs et maisons », un petit musée de Sologne fut installé en juillet 1955 dans les locaux de la mairie de Romorantin : il y est peut-être encore. On y déclinait le milieu naturel, l'apport humain, l'habitat, les techniques agricoles et artisanales, mais aussi la stratification sociale et ses incarnations, la vie quotidienne et les techniques. Bref, une écomuséologie à fondement interdisciplinaire.

\section{Patrimoine culturel, patrimoine naturel: une histoire mêlée}

C'est ainsi que m'est apparu, dès mes débuts, le métier d'ethnologue ruraliste: un domaine complexe, produit d'une histoire longue, lente mais non dépourvue d'accélérations et de coupures, composite et difficile à déchiffrer.

Durant le demi-siècle écoulé, les idées de base en la matière ont beaucoup évolué, les disciplines et moyens scientifiques à mettre à contribution se sont multipliés. Mais ce qui était présent depuis toujours et reste très important, ce sont les motivations non scientifiques qui pèsent sur la recherche, les intervenants politiques, comme les institutions parties prenantes: leur rôle a toujours été marqué et parfois prépondérant, comme on aura l'occasion de le montrer par la suite.

Mais, surtout, deux séries de faits ont marqué les dernières décennies. Les changements survenus, en matière technique, politique, économique, démographique - ce que l'on peut appeler l'action d'une histoire accélérée - ont agi sur les sociétés rurales, les ont altérées, transformées, densifiées, dotées de nouveaux moyens: l'équation homme-nature en a été profondément modifiée.

Parallèlement, la coupure entre patrimoine naturel et biens culturels, tranchée jusque dans les années 1950, allait s’atténuer. Jusqu’alors, les « parcs naturels », dispositifs majeur de préservation de la nature, étaient peu nombreux, on en trouvait aux États-Unis, en Suisse (dans les Grisons) : il s'agissait, selon la conception d'alors, de réserves naturelles, dans lesquelles, on laissait à ce qu'on appelait alors la nature le soin de conserver la nature, l'homme se bornant à clore et à protéger ces espaces. 
Dès les années 1960, la transformation de la France - qu'avait laissé entrevoir le livre prémonitoire de Jean-François Gravier Paris et le désert français dès 1947 - fut à la fois profonde et fortement perçue. L'exode rural s'accélérerait, la majeure partie de l'espace rural se trouvait quasi désertifiée. En même temps, on assistait à une expansion et à une densification des villes, à une croissance des banlieues, à la création de villes nouvelles, ce qui entraîna la création d'espaces naturels d'accompagnement, véritables « poumons verts » en même temps que de lieux de loisir pour les habitants. Ce fut l'invention d'un concept et d'une institution apparemment contradictoires au point de vue logique et par rapport à la pratique antérieure: le parc naturel régional. À noter que ce sont la fonction publique et l'appareil gouvernemental qui ont joué, en France, un rôle moteur dans ce domaine, comme d'ailleurs dans l'ensemble de la politique environnementale.

En même temps que l'on y créa, enfin, des parcs naturels au début des années 1960, des parcs régionaux furent organisés (Camargue, Armorique, Saint Amand) ou projetés (le Morvan, etc.), soit plus de dix en 1966.

Le moment fondateur vint, cette année-là, avec « Les journées nationales d'études sur les parcs naturels régionaux» qui se sont tenues à Lurs-en-Provence du 25 septembre au ler octobre (Collectif 1967). Organisées par un " groupe de travail inter-ministériel des parcs naturels régionaux » sous les auspices du Ministre délégué chargé du plan et de l'aménagement du territoire - alors Raymond Marcellin -, ces journées furent ouvertes par Olivier Guichard, délégué à l'aménagement du territoire et à l'action régionale.

Ce fut une grand-messe: des intervenants très variés - des hommes politiques, des administrateurs, des scientifiques, des techniciens, des architectes, des militants sociaux, des artistes, etc. - y présentèrent des communications, ou intervinrent dans les débats. Les thèmes mis en avant pour justifier la création des parcs naturels régionaux furent les nouveaux besoins des grandes métropoles, la présence d'importantes zones rurales abandonnées à revivifier et - le plus important, déjà - le patrimoine culturel et naturel. L'horizon balayé fut très vaste.

Il en résulta un volume, compte rendu intégral, établi d'après la sténotypie des interventions et des discussions, document brut mais intéressant et qui mériterait d'être réanalysé à la lumière des presque quatre décennies écoulées depuis.

Après J. Labasse (" Milieu naturel et géographie volontaire ») et P. Monbeig («Le paysage, élément dynamique, vivant et instable »), j'avais choisi d'y traiter de «L'espace rural: création de l'homme », ensemble de réflexions que je ne renie pas aujourd'hui. J'y soulignais la complexité et le dynamisme de ce nouvel objet « parc naturel régional », espace à conserver mais fait, dans sa majeure partie, par la main de l'homme et habité par celui-ci. En majorité rural, fait pour produire avant tout, cet espace est artificiel, fabriqué. Il est aussi et toujours un système en équilibre: entre homme et nature, entre processus naturels et activités humaines, entre campagnes et villes voisines. Fruit d'une longue évolution, au rythme et 


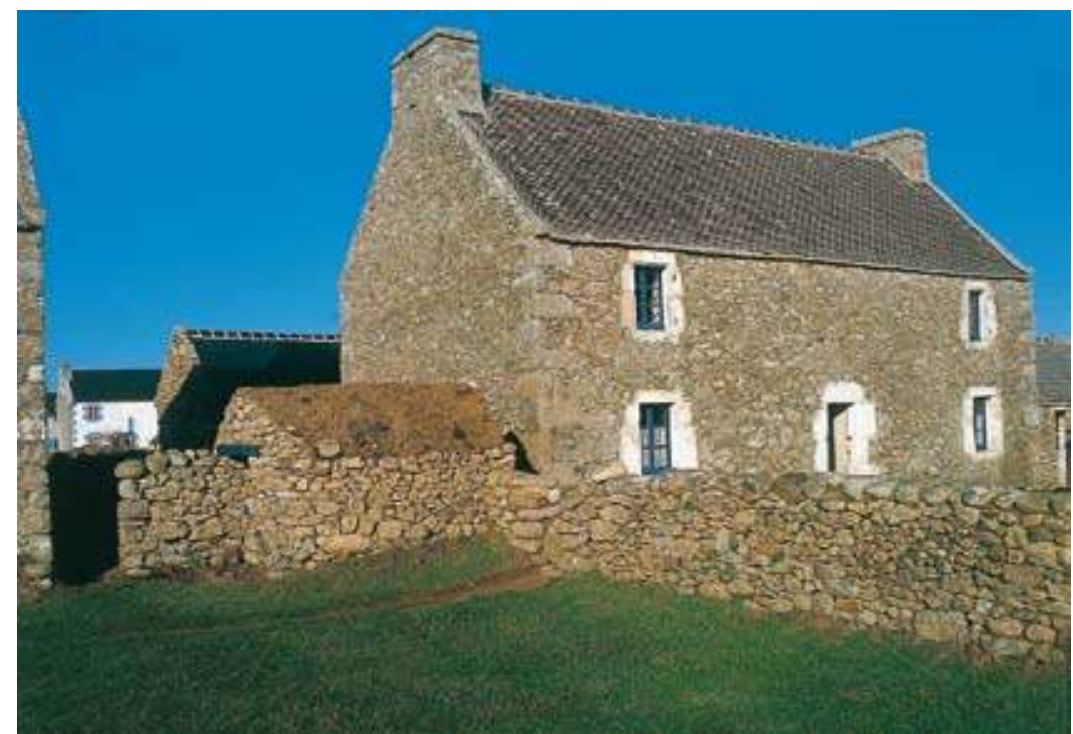

Écomusée d'Ouessant, Parc naturel régional d'Armorique, (c) Jean-Pierre Gestin. à la durée particuliers, cet espace est doublement aménagé, sur le plan technique d'une part, social et symbolique de l'autre. Son équilibre est fragile, sa dégradation, lorsqu'elle survient, est parfois irréversible.

Une autre intervention, mémorable à plusieurs égards, fut celle de G.-H. Rivière, créateur et conservateur en chef du musée national des Arts et Traditions Populaires (Chiva 1985). Alors que la France ne connaissait pas cette institution, courante en Europe depuis la fin du XIX siècle, que sont les musées de plein air, il traita de l'architecture rurale et proposa sa sauvegarde notamment par le biais des écomusées (dont le premier fut créé sur l'île d'Ouessant en 1966).

Revenant sur l'objet du colloque de Lurs, G.-H. Rivière parla explicitement de « parcs naturels et culturels » alors que Michel Parent, alors inspecteur des Monuments historiques et des sites et grand défenseur de l'architecture rurale, préconisait, en une formule syncrétique, la « conservation du site, du milieu humanisé et du monument culturel ». Excellente manière d'expliciter l'indissociabilité des patrimoines naturel et culturel!

Beaucoup plus tard - et je le mentionne pour souligner à quel point certaines personnalités atypiques, sans étiquette fonctionnelle ni autorité nominale, mais porteuses d'idées et de convictions fortes ont joué un rôle de premier plan en la matière - Jean Blanc, membre en 1966 du groupe de travail interministériel des parcs naturels régionaux, reviendra sur cette vision unitaire lors d'une rencontre conservationiste qui sera aussi une occasion manquée. Le colloque « Musées et sociétés » organisé en juin 1991 à Mulhouse et à Ungersheim, siège de l'écomusée d'Alsace, devait préparer la création d'une organisation distincte fédérant musées des sciences sociales et écomusées (cf. Barroso \& Vaillant (eds) 1993), création qui ne se concrétisa pas. Chargé d'un des rapports introductifs destinés à orienter les débats, Jean Blanc y récapitulera, à partir du moment de Lurs, moment fort d'une période allant de 1965 à 1972, une réflexion sur l'environnement comme « système dynamique ». Il y soulignera le caractère « complémentaire et solidaire » des parcs naturels régionaux et des écomusées, définis comme "musées spécifiques de l'environnement $»$. Et il conclura par une formule définissant les écomusées qui mérite d'être rappelée: « Ces approches ethno-écologiques intégrales bouleverseront les principes de la muséographie et même peut-être la définition classique de la culture ».

Pour revenir aux journées de Lurs, en sortiront la formule des parcs naturels régionaux, leur carte et leur regroupement en une « fédération ». Ceux qui participent à ce processus, comme ceux qui écrivent dans 
les publications de la Fédération des parcs naturels régionaux de France, sont souvent des amateurs éclairés ou encore viennent aussi bien des sciences de la vie que des sciences de la société. Il en résulte aussi une frontière désormais définitivement brouillée entre parcs naturels et parcs régionaux, comme entre institutions nationales et institutions régionales.

C'est par le biais de ce processus - et en particulier à propos des écomusées et des musées - que la notion de patrimoine allait acquérir en quelque sorte, droit de cité. Ce « concept nomade »-pour emprunter une catégorie logique d'Isabelle Stengers formulé dans de multiples champs disciplinaires, aussi bien naturels que culturels, allait s'imposer progressivement. Les années 1979-1980 allaient voir la création, au ministère de la Culture, d'une mission et d'un conseil, interministériel dans sa composition, du patrimoine ethnologique. Schématisé ainsi, ce processus institutionnel correspondait aux réflexions que quelques ethnologues métropolitains avaient conduites sur les changements nombreux, divers et profonds survenus dans l'après-guerre à la fois dans la société française et dans son ethnologie.

Le rapport Benzaid (1980) rend compte de ce processus et permet de le cristalliser. Deux évidences en sont le point de départ: la prégnance du concept patrimonial et l'axiome de l'indissociabilité du réel. On ne pouvait en effet dissocier les facettes de la réalité, pas plus qu'on ne pouvait dissocier les disciplines intervenant dans l'analyse de cette réalité, ou le rural inséparable de la ville comme de la société globale, ou encore le passé et l'avenir. On ne pouvait enfin séparer recherche et action culturelle de l'enseignement. On ne pouvait non plus dissocier la recherche sur les cultures, des institutions muséales et de protection des biens culturels.

Il n'y a pas lieu de reprendre ici les raisons qui expliquent l'introduction forte, insistante, de la notion de patrimoine ethnologique au cour de ce processus, à la fois analytique et institutionnel. On les trouvera exposées, ainsi que la façon dont cette construction conceptuelle s'est formée, dans l'étude bilan publiée par l'auteur de ces lignes en 1990, dans Encyclopaedia Universalis (cf. en particulier le paragraphe « Patrimoine: la notion et ses contextes » qui retrace la configuration notionnelle).

Le contexte de la société française de l'après-guerre était marqué à la fois par la juridisation et par l'émergence forte de l'idée de nature. Les années 1965-1970 furent celles d'une crise économique qui s'accompagna d'une crise de conscience sociale. On eut alors recours, pour comprendre ce qui se passait, a des démarches scientifiques qui pouvaient converger. En ethnologie, la diversité culturelle s'imposa comme perspective majeure: Claude Lévi-Strauss parlera d'« optimum de diversités». Les apports 


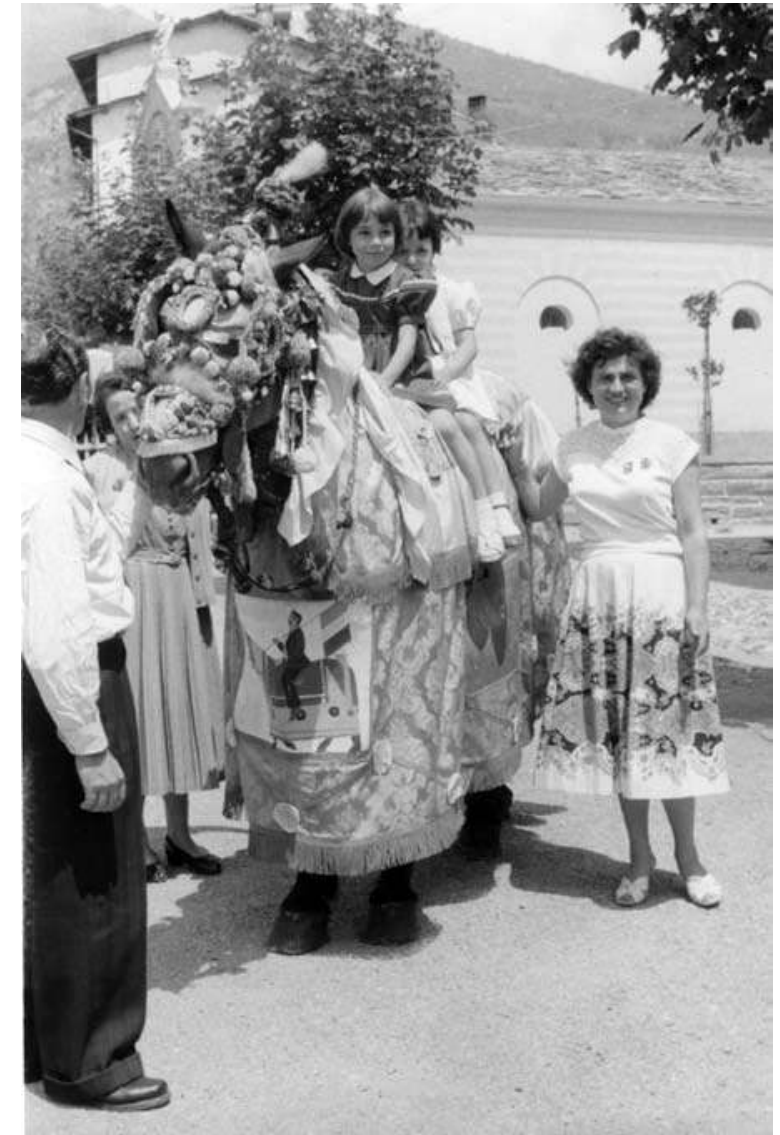

Enfants sur un mulet paré, fête d'été de la Saint Éloi, Tende, 1955, G. Fink. de l'agronomie, de l'économie, de la biologie génétique et particulièrement de la génétique des populations humaines (surtout les travaux sur les isolats), se combinèrent en une série d'enquêtes collectives et multidisciplinaires (notamment celles sur Plozévet en Bretagne et sur les Baronnies des Pyrénées).

Le patrimoine ethnologique, tel que nous l'avons défini à plusieurs reprises entre 1979 et 1990, se caractérise par sa diversité, son indétermination fonctionnelle, son mode de transmission lié avant tout à la parenté et à la dévolution héréditaire, par son évolution dans la longue durée, par son adaptabilité à des situations et à des usages non prévisibles dans l'immédiat. Mais surtout, l'homme, l'animal, le site sont partie intégrante du patrimoine ethnologique dont les composantes sont, pour certaines, redevables à des disciplines voisines de l'ethnologie. L'usage du concept de patrimoine ethnologique se fonde sur l'utilisation heuristique de la différence culturelle et du processus de diversification. C'est, nous semble-t-il, ce qui permet une lecture unifiée du patrimoine en un champ regroupant le culturel et le naturel, ces deux termes étant pris dans leur acception la plus large.

\section{Nature, technique, société: les logiques à l'œuvre}

On a, pendant longtemps, opposé la nature, facteur inerte, à la technique, facteur dynamique, surtout dans ses aspects modernes. Entre les deux, la société, facteur mou, quelque peu magique, pour autant qu'on s'en servait pour expliquer l'inexplicable dans la vie des campagnes. C'est aux facteurs sociaux que l'on avait, en effet, recours, pour éclairer tantôt les avancées, tantôt les reculs, ou pour échapper au dilemme inertie-progrès.

Aux interactions constantes réelles, entre ces trois ordres de faits, s'ajoutent une nécessaire lecture de leurs isomorphismes, de leurs correspondances ou distorsions, comme aussi les effets d'influence et de lecture sur chacun d'eux de l'indissociable rapport des villes avec les campagnes, de l'économie agricole avec l'économie générale, de la nature avec le fantasme urbain de la nature. 
Comment aborder ce thème qui a suscité une masse énorme d'écrits et de dits, d'idées, de passions, d'illusions, sinon par le biais de quelques notions simplificatrices? Le moment d'abord, en France comme dans le reste de l'Europe!

Les quarante ans, lourds de changements, qui ont suivi la seconde Guerre Mondiale nous ont conduits à une crise profonde et prolongée, dont on entrevoit à peine la sortie. Ses manifestations et son vécu ont affecté aussi bien l'économie que les formes de la vie sociale, les convictions intellectuelles, les idéologies, les modes de vie et de travail! L'État, la science, le progrès technique, en agriculture comme ailleurs, la ville au profit de laquelle les campagnes se sont vidées partout à un rythme accéléré, la ville en particulier, jadis lien d'ancrage de convictions motrices - tout cela a été mis en cause. En particulier - et ceci nous concerne directement - les revendications et aspirations liées à la nature - sont des signes qui ne trompent pas de cette profonde crise de nos sociétés européennes. La défense de la nature s'accompagne ainsi d'un refus multiforme, même s'il n'est que de principe - car on ne refuse pas le bien-être quotidien fruit du progrès - refus de la rationalité unique et univoque de la société industrielle. Véritable contre-culture propre aux périodes de transition, telle celle que nous venons de vivre, cette « priorité de principe accordée à la nature sur la culture » selon l'expression de Claude Lévi-Strauss, et qu'expriment les mouvements tournés vers la nature, traduit à chaque fois et à coup sûr une période de crise, de doute, de mutation. Nous voyons ainsi - et cela depuis bientôt deux décennies - la revendication d'un véritable « droit à la nature », la défense de celle-ci et la constitution de la valeur/nature en anti-valeur/technique (ou anti-valeur science, ou civilisation d'ailleurs...); ce qui traduit des changements essentiels survenus dans les esprits. C'est notamment ce qui a conduit à valoriser les savoirs naturalistes traditionnels détenus par les paysans, comme à multiplier parcs, musées, écomusées destinés à illustrer, à conserver, à perpétuer ces savoirs auxquels nous aurons à revenir. Ajoutons qu'au même titre que l'aspiration vers la nature, cette double crise - sociale (de conscience collective) et économique - se traduit dans le passéisme ambiant, dans le retour vers le passé qu'incarnent précisément les campagnes, les villages et les paysanneries - leurs modes de vie et leur savoir faire, leur architecture et leur civilisation matérielle, leurs paysages.

Rappelons, en regard de ces faits de mentalité, les quelques faits bruts, chiffrables qu'illustrent les transformations des campagnes européennes et de leurs rapports avec le reste de nos sociétés: forte diminution de la population agricole; forte croissance urbaine pendant longtemps, suivie 


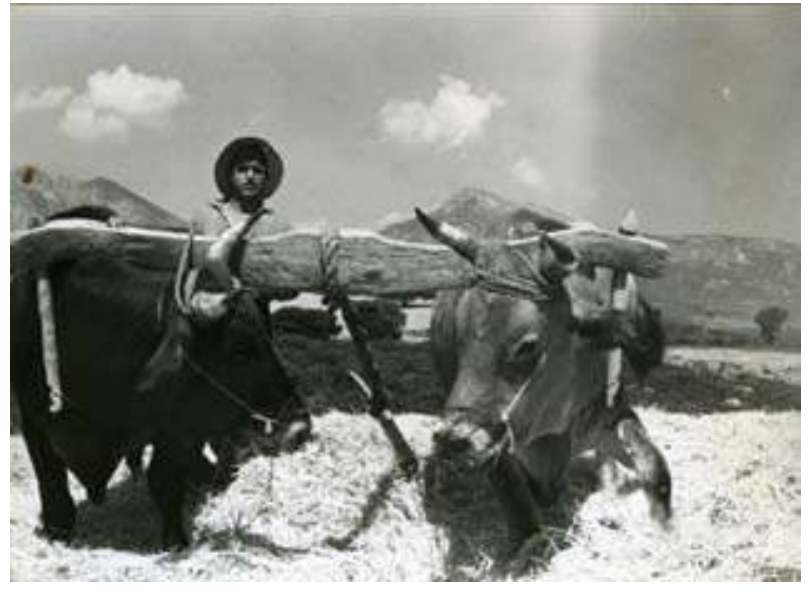

Battage de l'orge, Corse, 1956, I. Chiva. plus récemment, ici et là et en France notamment, d'un glissement des urbains vers la campagne: pour les départs en vacances, au vert, à la neige, à la mer, véritables exodes annuels; par l'expansion du phénomène des résidences secondaires, par l'installation de plus en plus fréquente dans les villages des retraités urbains mais aussi de nombreux actifs qui continuent à travailler à la ville. Substantiels, ces déplacements de population urbaine vers les campagnes ont de nombreuses conséquences sur le devenir de celles-ci, en particulier du fait que ces néo-ruraux interviennent souvent et activement dans la vie locale, allant jusqu'à une fréquente prise du pouvoir municipal.

Enfin, parallèlement au mouvement des hommes, il convient de souligner un autre fait dont les conséquences sont significatives dans la perspective qui est la nôtre, celle des sociétés rurales et de leurs rapports avec la nature et la technique. Pour des raisons variées - meilleure répartition de la croissance dans l'espace, création d'emplois dans des zones défavorisées, moindre coût de la main-d'œuvre rurale, volonté d'éloigner les usines du climat politique et social des grandes villes - nombre d'industries petites et grandes ont été établies en dehors des villes, rendant partiellement caduque la coïncidence - et la confusion - entre ville et industrie. L'aspiration des gens à de meilleures conditions de vie, dans un cadre physique et social plus humain, celui que proposent les campagnes, y a certainement joué un rôle. On aperçoit sans peine les conséquences de ce phénomène sur le comportement de la population rurale: conséquences variées allant de l'augmentation de la double activité, à la modification des mentalités et comportements économiques, en passant par le changement des rapports avec les techniques modernes. Deux mondes - celui, mental et social de l'atelier ultramoderne, celui de l'atelier agricole et pastoral, aux techniques et aux habitus traditionnels - se partagent ainsi de plus en plus les mêmes individus, coexistent dans une même biographie, avec les conséquences que l'on peut imaginer, et que l'on n'a pas encore assez étudiées. Ce qui nous conduit à aborder la manière dont il convient de déchiffrer notre équation et à éclairer le rapprochement des termes «nature, techniques et société ».

Avec leur esprit analytique, les sciences de l'homme ont apposé sur le sujet humain et sur son monde une grille qui découpe et nomme nature, technique, société mais aussi politique, religion, arts et autres futilités. Plus peut-être que dans d'autres domaines, ce découpage, même s'il postule des liens entre ces réalités observées mais aussi construites, omet ou relègue au plan des spéculations apparemment inutiles, une double constatation qui vaut pour qui veut aborder le monde rural.

Première évidence: c'est l'homme, en tant que sujet insécable, sujet producteur de sens, qui est au cour du monde, de ce monde, et, partout, de nos recherches. 
Seconde évidence: producteur de significations, l'homme et particulièrement l'homme des campagnes, porte en lui plusieurs logiques distinctives, parfois contradictoires, forgées au contact des autres hommes, de la nature, de l'histoire; et cette pluralité de logiques, dont le plus souvent il n'est pas conscient, s'exprime dans les différents ordres de choses qu'il façonne, soit particulièrement dans l'ordre de la nature, dans celui des techniques, dans celui de la vie sociale. Non, la rationalité moderne ne gouverne pas l'action de l'homme dans chacun de ces domaines: à la place, et c'est la leçon des sciences de l'homme, alternatives ou simultanées, cohérentes entre elles ou contradictoires, plusieurs logiques, toutes intelligibles, y sont à l'œuvre. Couplées en dichotomies simplificatrices, ou distinctes et divergentes, ces logiques spécifiques soustendent chacun des niveaux isolés artificiellement pour les besoins de la cause, sur chacun de nos trois plans, l'homme des campagnes, bien plus que l'urbain, se meut, intervient et juge selon des logiques spécifiques. Nature, technique, société fonctionnent chacune selon un ordre qui lui est propre, et proposent chacune des enchaînements d'actions, d'effets, de causalités. Leur superposition impose néanmoins une lecture simultanée de cet enchevêtrement compliqué par leurs différences profondes. Différence de temporalité et de rythme: que l'on songe à la longue durée qui est constitutive du paysage agraire et inscrite dans celui-ci; et en même temps aux cycles variables, aux rythmes généralement courts de la biologie des espèces végétales et animales; ou aux rythmes différents auxquels obéissent investissements fonciers d'une part, opérations de commercialisation de l'autre. Ces exemples pourraient être aisément multipliés! À la différence de temporalité et de rythme, s'ajoute, bien sûr, la différence d'échelle des phénomènes observables dans les trois ordres qui, par ailleurs, proposent des supports et des mécanismes de pensée hétérogènes. Ainsi, en est-il sur le plan technique, en schématisant, de la coexistence - et souvent de l'opposition - entre la pensée encore empirique - et qui le sera probablement toujours pour une part -à l'œuvre dans la pratique des agriculteurs et la pensée rationnelle, liée à ce que Placide Rambaud (1996) appelle la « technicisation à base scientifique » de l'agriculture. Ou encore de la coexistence, dans l'ordre de l'économique, d'une pensée et d'un comportement patrimoniaux - nous y reviendrons - opposés à la pensée et au comportement rentabilistes, guidés par la rationalité économique.

Peut-on supposer - et extraire - dans ces conditions de complexité, une configuration logique sous-jacente et commune aux trois faces de notre réalité: nature, technique, société? Question ultime, difficile, que l'on se contentera de poser, sans tenter d'y répondre.

Limitons-nous ici à illustrer rapidement notre propos: à savoir que, dans chacun de ces trois sous-univers du monde rural, deux ou plusieurs logiques sont à l'œuvre, tantôt complémentaires, tantôt antagonistes; et que tenir compte de ce pluralisme des logiques à l'œuvre permet de comprendre autrement, mieux, même les changements les plus actuels qui affectent les campagnes européennes. 


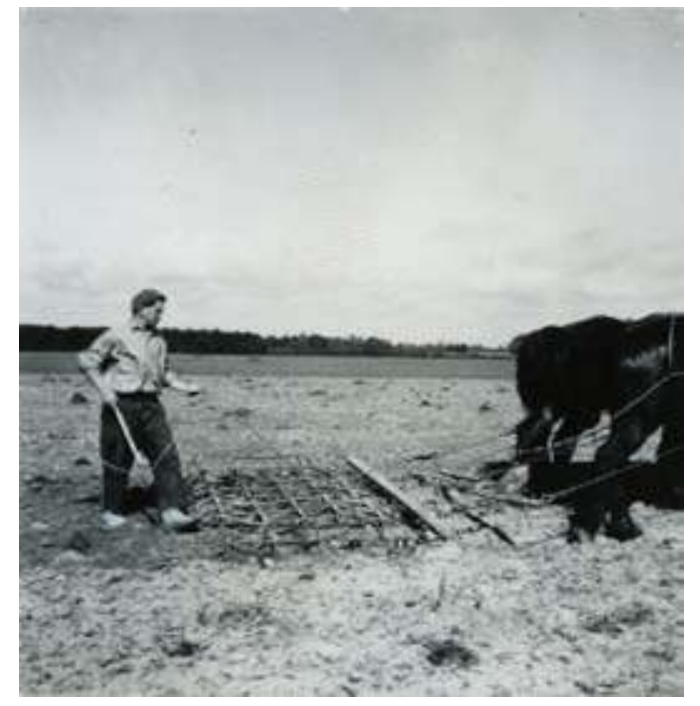

Hersage, St Viatre, Loir-et-Cher, 1951, I. Chiva.
Premier exemple: celui des comportements techniques. On commence à avoir une vue claire des savoirs naturalistes populaires: on perçoit de mieux en mieux leur étendue, leur précision - celle d'un véritable naturalisme empirique - mais aussi les logiques particulières, fort cohérentes, à l'œuvre dans leurs classifications. On met aussi de plus en plus en évidence la charge symbolique qui non seulement s'attache aux plantes et animaux, mais fait que leurs noms, leurs propriétés, leurs parentés permettent véritablement de comprendre comment les hommes qui possèdent ces connaissances pensent leur monde, leur travail, leur propre être. Or c'est un véritable tissu de connaissances, de fonctions métaphoriques, d'enchaînements, associations et causalités attribués, utilisés tacitement, qui sous-tend et conditionne encore dans une large mesure les actes techniques de l'agriculteur. C'est en fonction de cette logique empirique des qualités et des classements du monde naturel que traduit toujours et fidèlement un vocabulaire approprié, que l'homme de la terre accueille et comprend les propositions du technicien. Ses mots, son parler en sont pleins, comme l'ont montré nombre d'observations, et conditionnent la compréhension, des notions techniques qui lui sont proposées. À cela s'ajoutent les savoirs techniques proprement dits - ceux du sol et du climat, des outils et de leur fonctionnement, de leur efficacité - véritables savoirs professionnels empiriques, produits d'une pratique et d'une tradition plus ou moins longues, archaïques ou de pointe, et toujours basés sur la connaissance et la transformation de la nature. La technique moderne ne pénètre donc jamais chez l'agriculteur en terrain vide, dans un esprit vierge. Les savoirs, les notions, les pratiques avec lesquels elle doit composer sont en outre liés à des dispositifs sociaux, à des traits plus ou moins durables de la vie sociale qui conditionnent les réactions des individus à la novation technique comme à la crise économique. C'est pourquoi il est important, comme le dit Jean-Pierre Darré (1985), un des observateurs les plus fins de l'interaction entre agriculture et modernité, de comprendre réellement « les savoirs et pratiques des praticiens autrement que comme périphéries altérées de la vérité scientifique contemporaine ». C'est pourquoi il est important de reconnaître leur juste qualité - celle d'une vraie logique, d'un système cognitif véritable -, les notions, procédés mentaux et techniques propres aux praticiens de l'agriculture.

Se poser la question des connaissances empiriques de l'agriculteur, des normes qui guident celui-ci dans le domaine de la technique, s'interroger sur la source de la légitimité de ces normes face à la logique technicoscientifique, reconnaître le caractère complexe de cette pensée empirique, pratique, n'est pas une spéculation gratuite. C'est se donner les moyens, en évaluant la plus ou moins grande plasticité de ce système de penséeaction, de pronostiquer l'aptitude des agriculteurs actuels au choix et à 
l'adaptation, en un mot à une stratégie volontaire. Reconnaître l'importance des savoirs empiriques, le tissu complexe qui unit entre elles pratiques, notions, images, valeurs, conduit à comprendre selon quels mécanismes l'agriculteur et l'éleveur, ou celui qui est les deux, savent prévoir et absorber les risques du métier, intégrer les novations, jouer avec la gamme des produits et des probabilités du marché.

Se poser ainsi, de cette manière, la question des rapports entre nature, technique et société revient non plus à postuler le principe d'un lien entre ces trois ordres de réalités, mais à démontrer concrètement le fonctionnement social du travail agricole et ses rapports avec la production sociale des normes et valeurs, ainsi que les liens entre transformation des activités techniques et augmentation des besoins de communication. Ainsi les logiques à l'œuvre dans le domaine technique passent-elles nécessairement par le sens des mots.

Observons un autre exemple de la dualité des logiques à l'œuvre, cette fois-ci dans l'ordre des comportements sociaux et économiques, et notamment à propos de la terre, bien qu'il forme le noyau de ce que l'on nomme le patrimoine foncier. Accumulation du capital, production et consommation de biens, activités de service sont autant de comportements économiques qui entrent dans le champ de la rationalité et du calcul proprement économiques et occupent des parts spécifiques dans le tableau des ressources et des dépenses des ménages des différentes couches sociales à la ville et à la campagne. De ce point de vue, on peut déterminer bien entendu un profil économique particulier des ménages d'agriculteurs dont les revenus sont avant tout ceux d'exploitations individuelles et dont la dépense privilégie l'épargne, affectée principalement au financement de leurs moyens de travail. Mais aux biens économiques et particulièrement au capital proprement dit, s'opposent les biens patrimoniaux, et parmi ceux-ci principalement la propriété foncière agricole qui, pour les familles, a fonction de patrimoine. Or la valeur que l'on attache aux biens patrimoniaux n'est que très partiellement d'ordre économique. Ils circulent principalement, en vertu de la dévolution héréditaire, soit par héritage soit par dotation, selon des schémas parallèles aux réseaux d'alliances matrimoniales et selon des stratégies spécifiques, obéissant à des normes différentes sinon parfaitement contraires à celle de la rationalité économique. Maintenir, étendre et transmettre des biens porteurs de valeurs sociales, de prestige et de continuité en même temps que de valeur vénale, sont les motivations principales des comportements patrimoniaux. Conçu de la sorte, le patrimoine remplit des fonctions nombreuses et hétérogènes, irréductibles les unes aux autres, nettement différentes des fonctions économiques proprement dites. « La pluralité des fonctions attribuées au patrimoine dépend de la pluralité des rôles distingués par les agents » écrit Jean Cuisenier. Pour lui, le patrimoine étant « l'ensemble des biens qu'il convient de conserver en en faisant fructifier l'héritage, le comportement patrimonial apparaît comme ayant pour norme primordiale le souci de préserver et d'étendre ces biens », c'est-à-dire avant tout des motivations de continuité sociale, de statut, de prestige, d'identité familiale. Ce sont là autant d'éléments qui permettent à la société, aux groupes familiaux qui la composent, de se perpétuer, au-delà 
des finalités prévisibles et des rentabilités immédiates qui caractérisent les comportements économiques non patrimoniaux. Et n'est-ce pas ici même, lors du colloque de 1979, à propos de la forêt, que Jacques de Montgolfier soulignait que « les biens patrimoniaux sont ceux dont tous les emplois ne sont pas prévisibles dès maintenant », ce qui donne une bonne mesure de la distance entre les deux logiques que je viens d'opposer, une bonne idée de la nature de la différence entre les comportements correspondants (Montgolfier \& Bertier 1980).

On pourrait, au même titre, invoquer d'autres couples de logiques simultanément à l'œuvre dans le fonctionnement du monde des campagnes, mais ce serait allonger indûment le propos. Contentons-nous, pour finir, de mentionner l'opposition entre logique productiviste et logique conservatrice dans l'exploitation des ressources naturelles. Éviter l'épuisement de la terre, la dégénérescence des espèces, l'épuisement des espaces boisés et non cultivés, impose l'aménagement durable du milieu et freine la recherche de la productivité maximale. C'est là l'exigence principale qui aura présidé au processus de longue haleine et continu ayant permis la création des espaces ruraux aménagés. Et ce processus doit être poursuivi, car si l'on s'arrête, c'est la régression, la dégradation du paysage. Car ces espaces ruraux constituent, de plus, des systèmes en équilibre, dans lesquels se lit le souci inconscient mais fort qu'a toute société agraire de conserver la nature, tout en permettant à l'homme d'en vivre, en visant le maximum de sécurité et non pas le maximum de rentabilité.

Et c'est peut-être cette logique particulière qui permettra à ceux qu'elle habite et qui ont su instituer et respecter un certain équilibre entre l'homme et la nature, d'assurer la sauvegarde des paysages créés par la main de l'homme, au moment même où le gel et la friche sociale les menacent gravement.

Car un paysage aménagé, exploité qui se dégrade, c'est beaucoup plus grave qu'une nature non encore défrichée. L'abandon - comme la désertification - conduit à des processus de dégradation irréversibles. Et c'est l'indispensable sauvegarde de ces espaces en équilibre instable que seuls peut-être, la logique conservationiste, les savoirs naturalistes et techniques empiriques et des motivations, des attachements irraisonnés de l'homme de la terre à ce qui l'entoure permettront d'assurer.

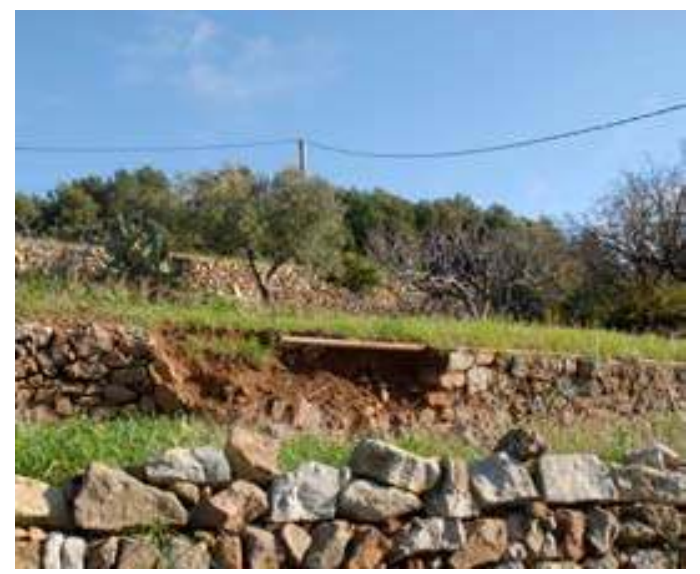




\section{RÉFERENCES}

Barroso Eliane \& Emilia Vaillant, (eds), 1993, Musées et sociétés. Actes du colloque Mulhouse-Ungersheim, juin 1991. Paris, Direction des musées de France.

Benzaild, Redjem, 1980, L'Ethnologie de la France. Besoins et projets. Paris, La Documentation française.

Collectif, 1967, Les Journées nationales d'études sur les parcs naturels régionaux. Lurs-en-Provence, septembre 1966. Paris, La Documentation française.

Collectif, 1995, Patrimoine culturel, patrimoine naturel. Colloque 12 et 13 décembre 1994. Paris, La Documentation française.

Chiva, Isac, 1990, « Le patrimoine ethnologique: l'exemple de la France » in Encyclopaedia Universalis Symposium, Paris: 229-241.

Chevallier, D., Chiva, I., \& Dubost, F., 2000, « Linvention du patrimoine rural » in D. Chevallier (ed.), Vives campagnes. Paris, Ed. Autrement: 11-55.

Chiva, Isac, 1977, «Les changements et tendances manifestés durant les vingt dernières années dans la société française » in Colloque sur les implications psycho-sociologiques du développement de l'industrie nucléaire. Paris, SFEN : 14-25

Chiva, Isac, 1985, «Georges Henri Rivière ou un demi siècle d'ethnologie en France », Terrain 5: 76-83.

Chiva, Isac, 1994, Une politique pour le patrimoine culturel rural. Rapport présenté à Monsieur Jacques Toubon, Ministre de la Culture. Paris, La Documentation française.

Chiva, Isac, 1997, « Le patrimoine rural » in Pierre Nora (ed.), Science et conscience du patrimoine. Paris, Fayard: 227-231.

Darré, Jean-Pierre, 1985, La Parole et la technique. Paris, L'Harmattan.

Gravier, J.-F., 1947, Paris et le désert français, décentralisation, équipement, population. Paris, Le Portulan.

Levi-Strauss, Claude, 1952, Race et histoire. Paris, Unesco.

Levi-Strauss, Claude, 1983, Le Regard éloigné. Paris, Plon.

Montgolfier, J. de, \& Bertier, P., 1980, « La Forêt méditéranéenne » Forêt méditerranéenne I(1-2).

Rambaud, Placide, 1996, Les Fondements de l'Europe agraire. Textes présentés par Rose-Marie Lagrave et Monique Vincienne. Paris, L'Harmattan. 
Des hommes, des techniques et des paysages 

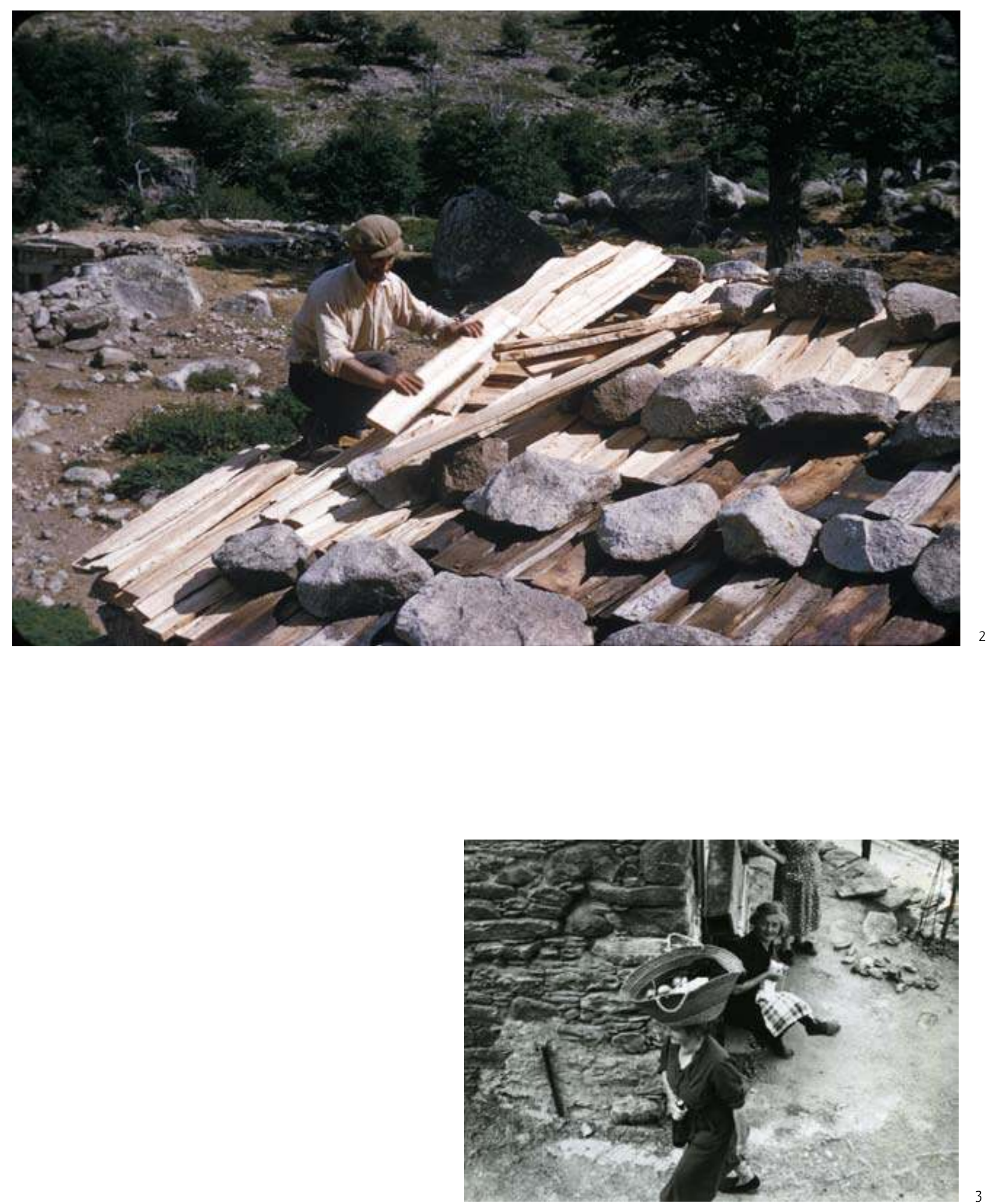

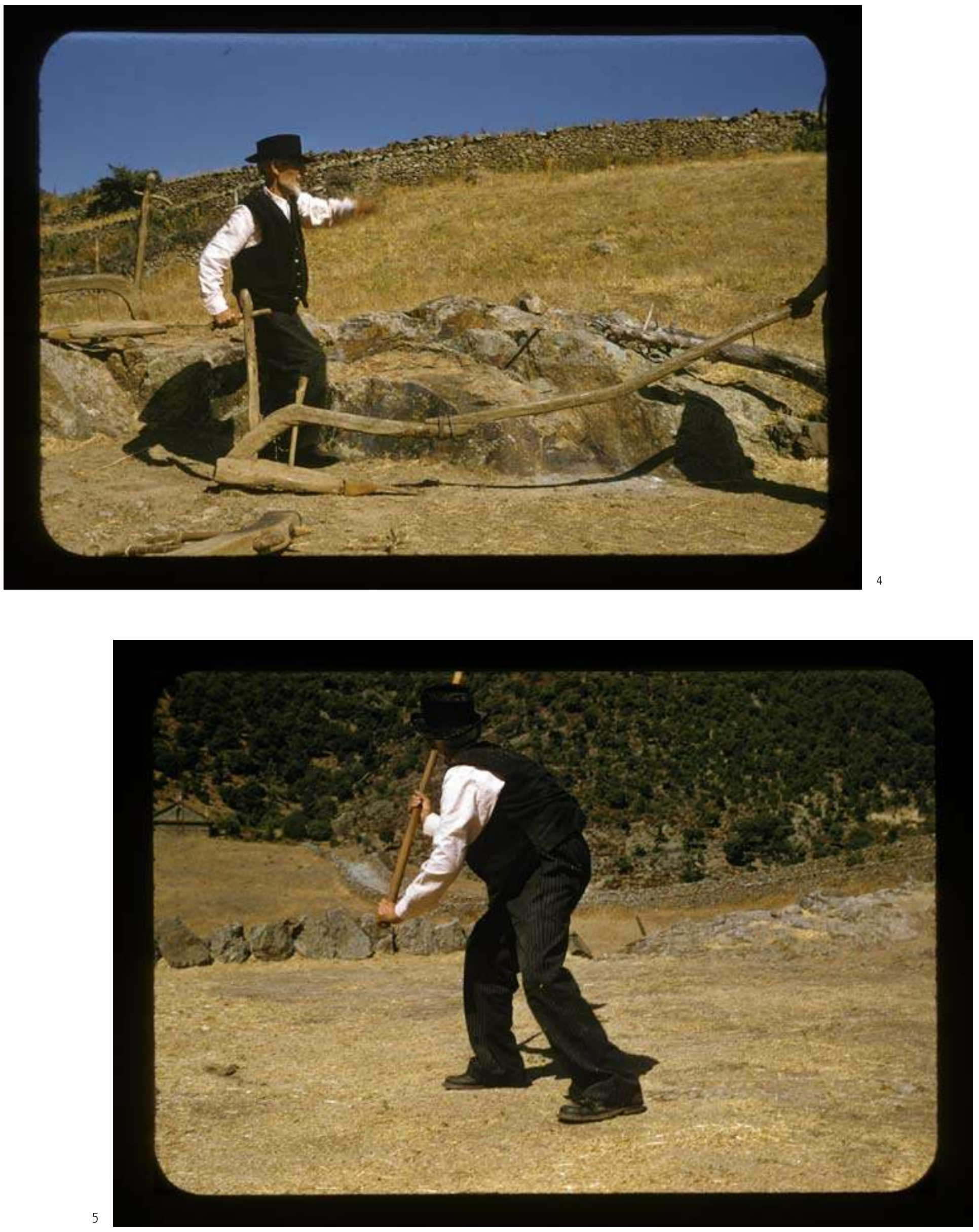


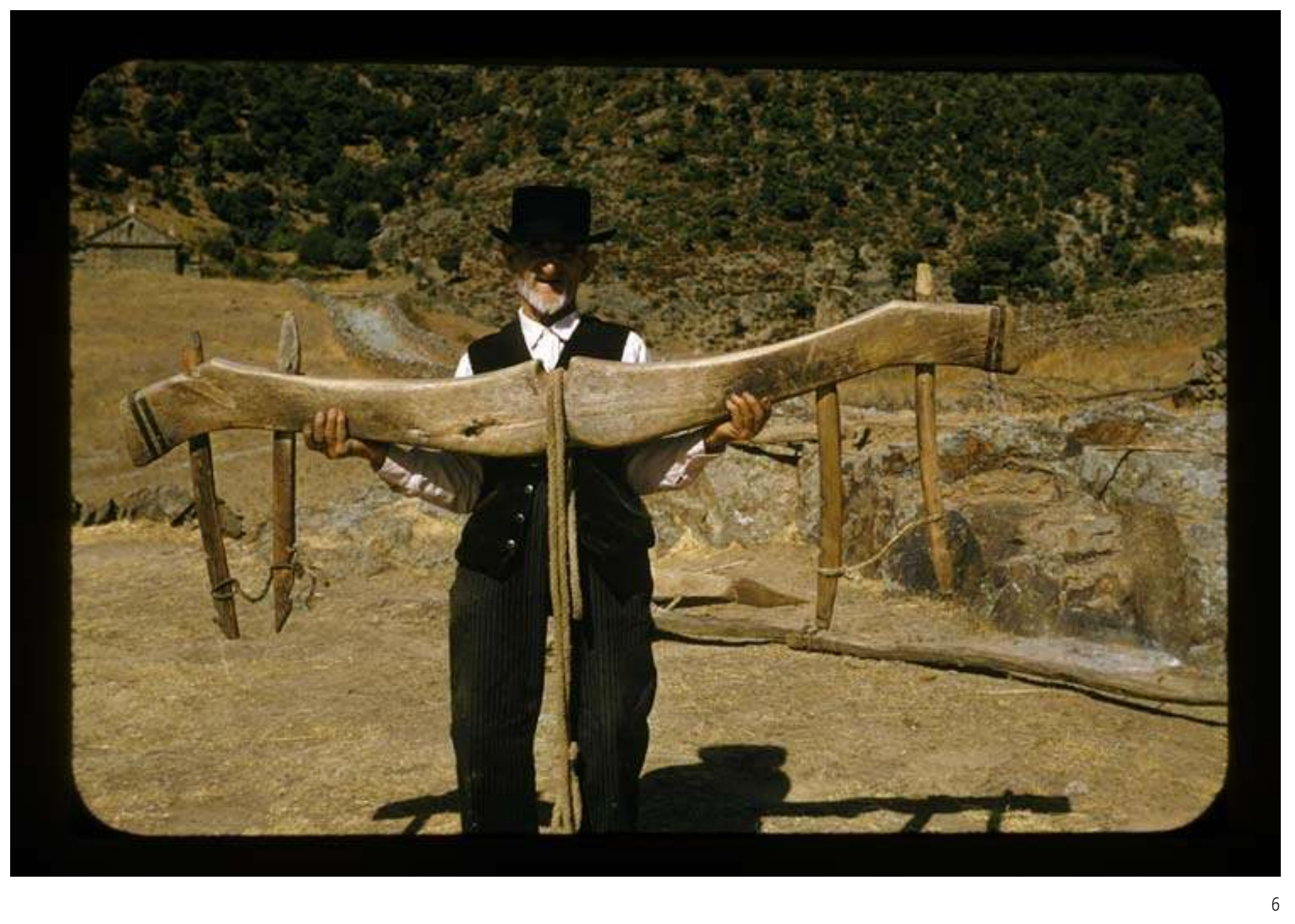



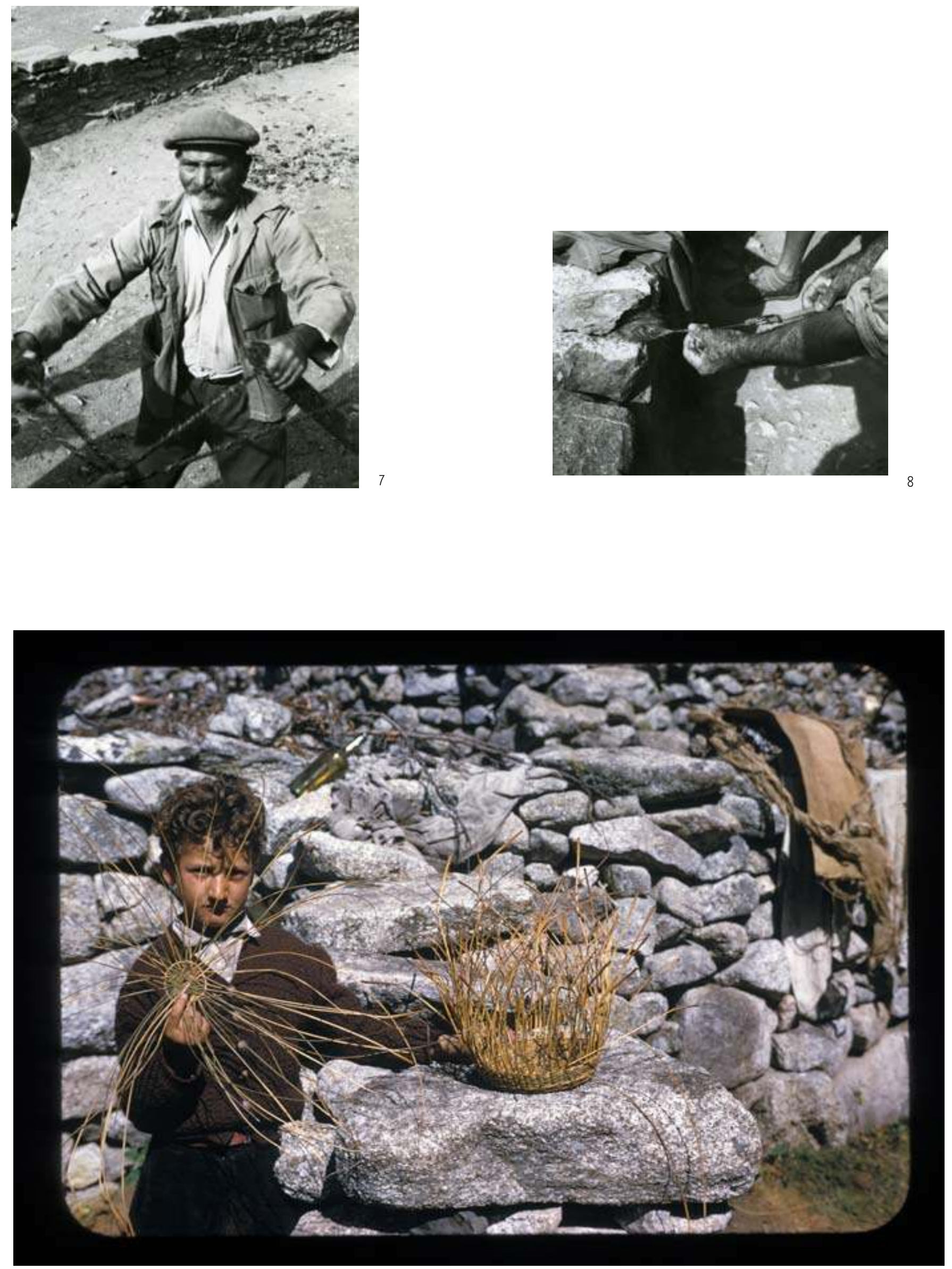


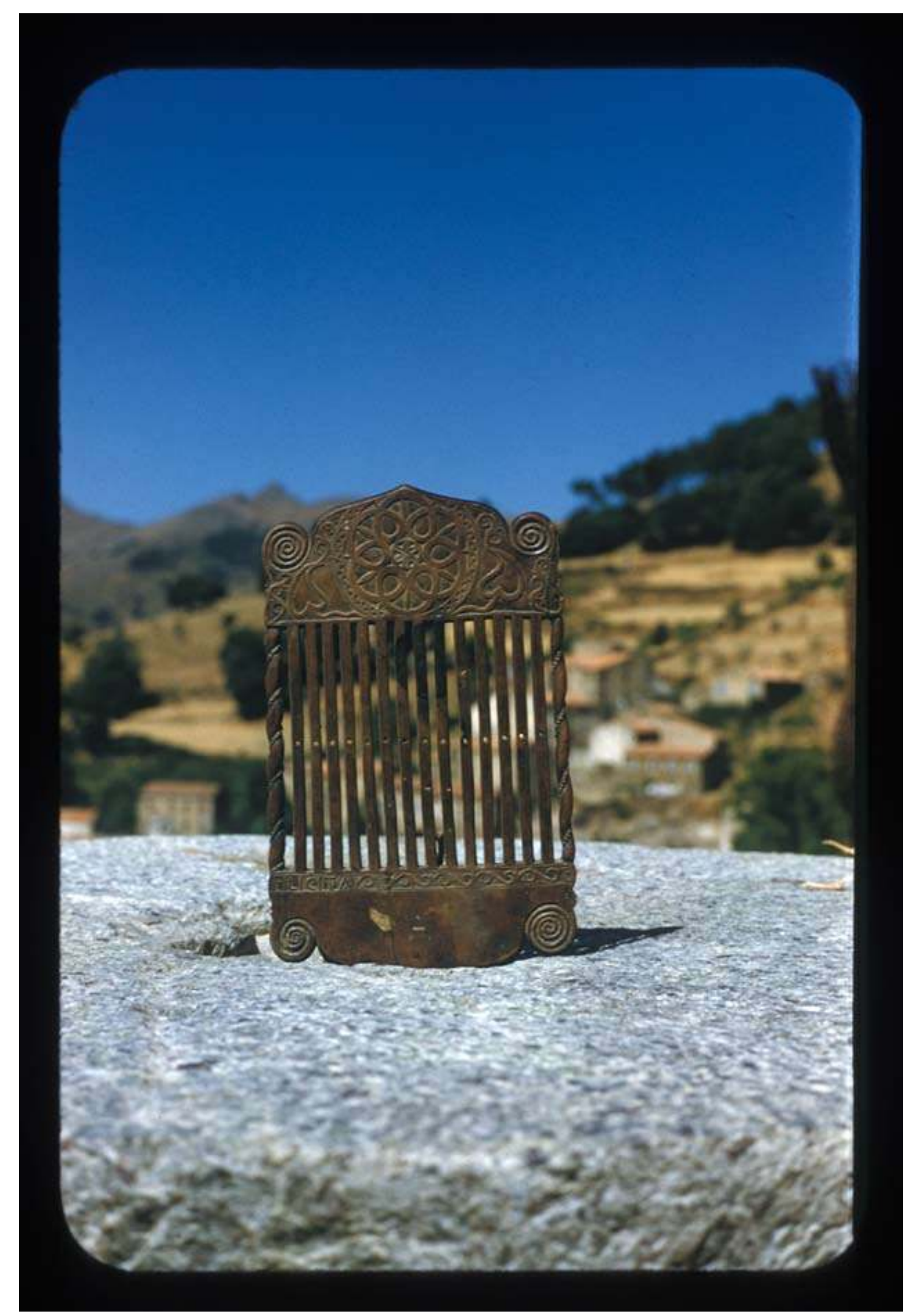



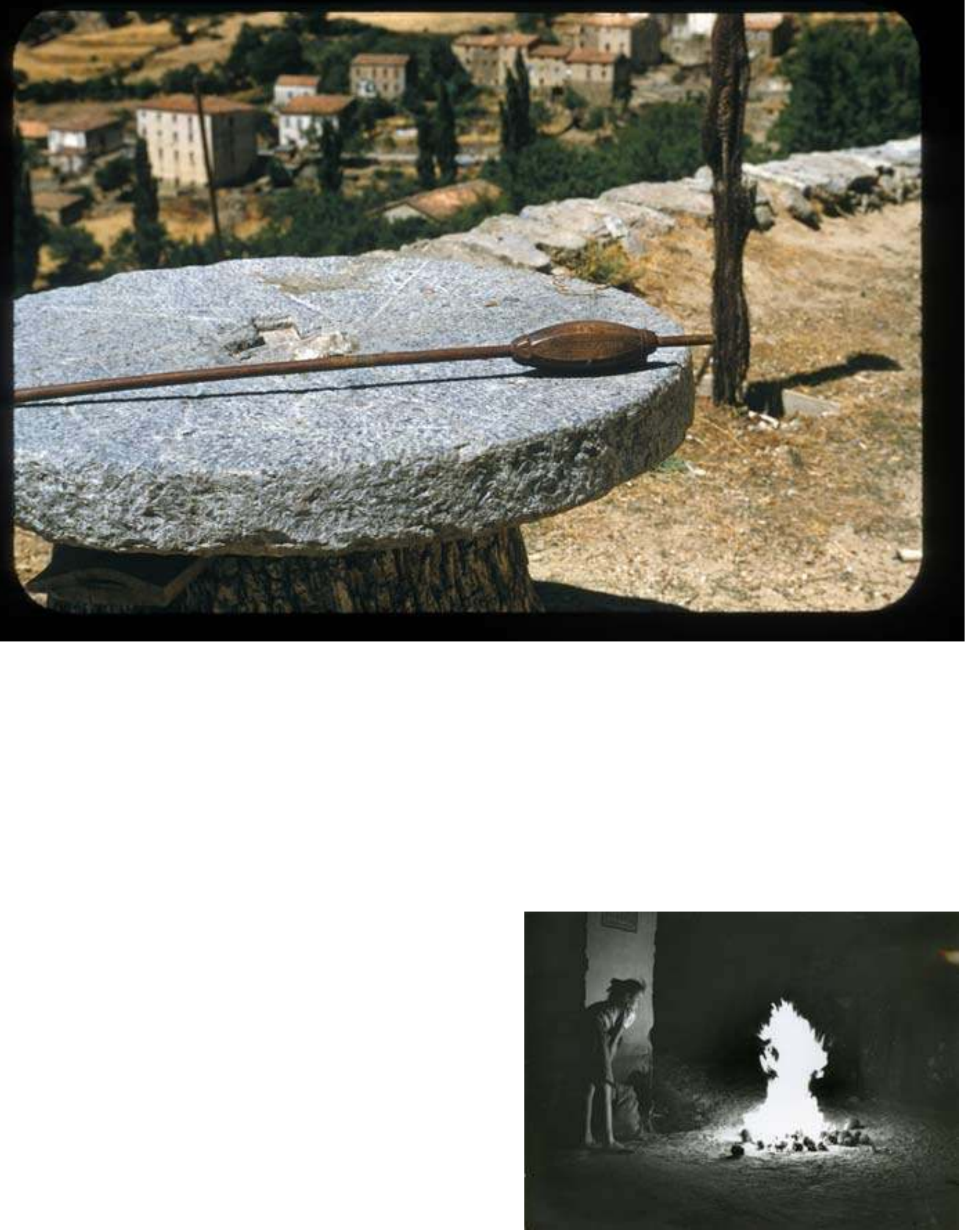


\section{LÉGENDES}

Archives Chiva, Laboratoire d'anthropologie sociale, Collège de France.

1. Tralonca, vallée au matin.

2. Moltifao, M. Ange-François Moltérini présente sa charrue en position de labours.

3. Moltifao, M. Ange-François Moltérini montre le maniement de la pelle à vanner sur l'aire à battre.

4. Moltifao, M. Ange-François Moltérini présente le joug.

5. Casamacioli, M. Lucciani remplace les bardeaux de la bergerie.

6. Portage sur la tête.

7. Cap Corse, tressage de cordes en poil de chèvre.

8. Casamacioli, enfant présentant paniers.

9. Olmi Capella, peigne à carder.

10. Olmi-Capella, quenouille.

11. Azzana, maison Richet, feu de protection devant les maisons, 31 juillet 1956.

12. Route de Tralonca vers Corte, incendie de maquis.

(Toutes les photos sont de l'auteur, 1956.)

Photo d'ouverture : Castagniccia, Corse, F. Joulian, 2008. 


\section{RÉSUMÉ}

Sciences de l'homme et patrimoines naturels. Quelques jalons historiques et théoriques. En France - et en Europe d'ailleurs - il y a, dans l'histoire du patrimoine, des « avant » et des « après »: les parcs naturels régionaux ont été lancés à l'occasion des "journées nationales d'études" de Lurs en Provence tenues en septembre 1966. Comme il y a un "avant" de la réflexion sur la notion de patrimoine, concept nomade qui prendra pied progressivement en biologie, en économie, en technologie, en ethnologie notamment, avant de trouver une formulation extensive et dynamique en 1994 avec le rapport "Une politique pour le patrimoine culturel rural", avant aussi d'aboutir à l'actuel programme "Patrimoine rural" piloté par le ministère de l'Agriculture. Cette histoire complexe, déjà ancienne mais toujours en cours, se lit à la fois en termes de domaines scientifiques, d'institutions, de dynamique sociales, de rapports changeants entre homme et nature, en particulier tels que les expriment les processus à l'œuvre dans la création et la transformation de l'espace rural.

\section{ABSTRACT}

Human Sciences and Natural Heritage: Some Historical and Theoritical Milestones. In France - and in Europe there is a turning point in the history of cultural heritage, a « before » and an « after » the moment when regional natural parks were launched, in a colloquium in Lurs en Provence in September 1966. Just as there is a « before » in the reflection on the notion of heritage, a nomad concept that moved from biology to economy, technology then ethnology before being formulated extensively and dynamically in 1994 in the report entitled "A Policy for Rural Cultural Heritage" and becoming the present "Rural Heritage" program run by the Ministry of Agriculture. This complex history, old already but still continuing, can be read in terms of scientific fields, of institutions, of social dynamics, of changing relations between humans and nature, particularly as expressed in the processes creating and transforming rural space.

\section{MOTS CLÉS}

Sociologie rurale, patrimoine naturel, paysage, techniques agraires, histoire des institutions, écomusée.

\section{KEYWORDS}

Sociology, natural and cultural heritage, landscape technologies, history of institutions, ecomuseum. 